\title{
An axiomatic characterization of the Slater rule
}

Citation for published version (APA):

Can, B., Pourpouneh, M., \& Storcken, T. (2021). An axiomatic characterization of the Slater rule. Social Choice and Welfare, 56(4), 835-853. https://doi.org/10.1007/s00355-020-01305-8

Document status and date:

Published: 01/05/2021

DOI:

10.1007/s00355-020-01305-8

Document Version:

Publisher's PDF, also known as Version of record

Document license:

Taverne

Please check the document version of this publication:

- A submitted manuscript is the version of the article upon submission and before peer-review. There can be important differences between the submitted version and the official published version of record.

People interested in the research are advised to contact the author for the final version of the publication, or visit the DOI to the publisher's website.

- The final author version and the galley proof are versions of the publication after peer review.

- The final published version features the final layout of the paper including the volume, issue and page numbers.

Link to publication

\footnotetext{
General rights rights.

- You may freely distribute the URL identifying the publication in the public portal. please follow below link for the End User Agreement:

www.umlib.nl/taverne-license

Take down policy

If you believe that this document breaches copyright please contact us at:

repository@maastrichtuniversity.nl

providing details and we will investigate your claim.
}

Copyright and moral rights for the publications made accessible in the public portal are retained by the authors and/or other copyright owners and it is a condition of accessing publications that users recognise and abide by the legal requirements associated with these

- Users may download and print one copy of any publication from the public portal for the purpose of private study or research.

- You may not further distribute the material or use it for any profit-making activity or commercial gain

If the publication is distributed under the terms of Article $25 \mathrm{fa}$ of the Dutch Copyright Act, indicated by the "Taverne" license above, 


\title{
An axiomatic characterization of the Slater rule
}

\author{
Burak Can ${ }^{1} \cdot$ Mohsen Pourpouneh $^{2}$ D $\cdot$ Ton Storcken $^{3}$
}

Received: 17 December 2018 / Accepted: 22 November 2020 / Published online: 5 January 2021

(C) The Author(s), under exclusive licence to Springer-Verlag GmbH, DE part of Springer Nature 2021

\begin{abstract}
Despite being a well-studied decision rule, the Slater rule has not been analyzed axiomatically. In this paper, we show that it is the only rule which is unbiased, monotone, tournamental, tie-breaking, and gradual. Thereby we provide a characterization of it for the first time. We also show these axioms to be logically independent.
\end{abstract}

\section{Introduction}

The well-known Slater rule captures the pairwise comparison idea discussed in Slater (1961). The rule chooses those linear orders (strict rankings) that are closest to the pairwise strict majority tournament. Here the concept of closeness is defined in terms of the Kemeny distance between the tournament and a given linear order. ${ }^{1}$ There has been a stream of literature on the analysis of the Slater rule both as a (collective) preference correspondence and as a (collective) choice correspondence. The preference correspondence chooses the linear orders as described above, where the choice correspondence chooses the tops of these linear orders.

For instance, Moulin (1986) and Laffond and Laslier (1991) analyze the rule as a choice correspondence. Meskanen and Nurmi (2006) and Nurmi (2002) discuss the rule within the context of distance from a consensus state. Baigent and Klamler (2003) exposes a comparison with the transitive closure rule while Klamler (2004)

\footnotetext{
${ }^{1}$ See Kemeny (1959) and Can and Storcken (2018).

Mohsen Pourpouneh

mohsen@ifro.ku.dk

Burak Can

b.can@maastrichtuniversity.nl

Ton Storcken

t.storcken@maastrichtuniversity.nl

1 Department of Data Analytics and Digitalisation, Maastricht University, Maastricht, The Netherlands

2 Department of Food and Resource Economics (IFRO), University of Copenhagen, Copenhagen, Denmark

3 Department of Quantitative Economics, Maastricht University, Maastricht, The Netherlands
} 
compares it to other pairwise rules such as the Kemeny and the Dodgson rules. In terms of the computational efficiency of the Slater rule (both as a choice correspondence and a preference correspondence) see also Hudry (2010) and Endriss and de Haan (2015).

This paper contributes to the aforementioned far from exhaustive list of existing literature by proposing a novel axiomatic characterization of the Slater rule as a collective preference correspondence, i.e., a rule that chooses sets of linear orders as outcomes. To that end, we introduce five conditions for preference rules: being unbiased, tournamental, monotone, tie-breaking, and gradual.

As a normative condition being unbiased requires that at uniform profiles, where all possible preferences are reported the same number of times, the outcome is that of a rule treating all alternatives equally. Many rules satisfy this condition. Being tournamental is standard. It means that the outcome is based on the pairwise strict majority tournament. It is the equivalent of the $\mathrm{C} 1$ condition for choice correspondences introduced by Fishburn (1977). Being tournamental is not satisfied by scoring rules, but besides the Slater rule there are other rules which are also tournamental, e.g., the Kemeny rule, the Copeland rule $^{2}$ and the Miller rule. ${ }^{3}$ The monotonicity condition, in the framework of choice functions, i.e., single valued choice correspondences, often leads to impossibility theorems. ${ }^{4}$ In the framework of preference correspondences, however, it is shown in Can and Storcken (2013) to be one of the characterizing conditions of the Kemeny-Young preference correspondence. Being monotone requires that if an agent changes (updates) her preference towards one of the preferences in the outcome, then the new outcome should still contain this preference and possibly more preferences but none that were not present before this update.

Finally, we introduce two new conditions tie-breaking and being gradual. Consider a preference profile with a pairwise tie on two alternatives. The former condition requires that a minimal change from this tie leads to a resolute outcome between these two alternatives. Meaning that in all outcome preferences the same strict order between these two alternatives is present. As such a resolution might already be present before the change in the profile, being gradual imposes that not at all new outcome preferences the order between these two alternatives is reversed. Many rules satisfy both these conditions. Among these rules are for instance the Copeland rule, the Kemeny rule, and the Borda rule. Together, these two conditions resonate with that of Baigent (1987), which proposes that a small change in the profile should also be represented by a small change in the outcome. Here, however, only changes from specific profiles are considered and changes are measured in terms of the Kemeny distance.

\footnotetext{
2 Copeland (1951).

3 The rule introduced in Miller (1980) takes all linear extensions of the cover relation. Here at a profile $p$ an alternative $a$ covers alternative $b$, if for all alternatives $x$ we have that $b$ beats $x$ in pairwise comparison at $p$ implies that $a$ beats $x$ in pairwise comparison.

${ }^{4}$ Muller and Satterthwaite (1977) show the equivalence of strategy proofness and strong positive association hence making the connection to the impossibility results of Gibbard (1973) and Satterthwaite (1975). Brandt et al. (2016) also introduces a monotonicity condition for tournaments only. We provide a detailed comparison between this condition and our framework in the Appendix.
} 
The paper is organized as follows. In Sect. 2, we formalize some basic notions and the model. Section 3 is on the characterizing conditions. In Sect. 4 we show how properties for preference rules translate to properties for tournament correspondences, assigning sets of preferences to tournaments. Section 5 discusses that the Slater rule is the only rule that satisfies these conditions simultaneously. Section 6 discusses the independence of the conditions used and concludes the paper. The appendix relates the monotonicity condition used here to a well-known monotonicity condition for tournament correspondences.

\section{Notational framework}

\subsection{Model basics}

We take $\mathbb{N}=\{1,2, \ldots\}$ the set of natural numbers as the set of potential agents. A set of (concerned) agents with cardinality $n$ is now $N=\{1, \ldots, n\}$, i.e., the first $n$ elements of $\mathbb{N}$. Let $A$ denote the finite set of $m$ alternatives with $m \geq 3$. The cardinality of an arbitrary (finite) set $S$ is denoted by $|S|$.

Let $\mathbb{L}$ denote the set of all possible linear orders over $A$, i.e., irreflexive, weakly complete, asymmetric and transitive binary relations on $A .^{5}$ For a generic $R \in \mathbb{L}$, and any two alternatives $a, b \in A,(a, b) \in R$ is interpreted as $a$ is ordered above $b$ at $R$. Note because of asymmetry of $R$ the latter can only hold for distinct alternatives $a$ and $b$. To simplify notation, we write $a b \in R$ instead of $(a, b) \in R$. Similarly, $R=a b c$ denotes a full ranking where $R=\{a b, a c, b c\}$. Further, notations like $a_{1} a_{2} \ldots a_{m}=R$ have the obvious meaning. Let $-R=\{y x: x y \in R\}$ denote the reverse of a linear order $R$.

Consider a set of agents $N$. A (preference) profile $p \in \mathbb{L}^{N}$ assigns to every agent $i \in N$, an element of $\mathbb{L}$, where $p(i)$ refers to the preference of agent $i$ at profile $p$. It therewith models a combination of individual preferences over agent set $N$. We call profile $p \in \mathbb{L}^{N}$ uniform if all linear orders in $\mathbb{L}$ are reported an equal number of times, i.e., for all $R, R^{\prime} \in \mathbb{L}$

$$
|\{i \in N: p(i)=R\}|=\left|\left\{i \in N: p(i)=R^{\prime}\right\}\right| .
$$

Let $N_{1}=\left\{1, \ldots, n_{1}\right\}$ and $N_{2}=\left\{1, \ldots, n_{2}\right\}$ be two sets of agents. Let $q \in \mathbb{L}^{N_{1}}$ and $r \in \mathbb{L}^{N_{2}}$ be two profiles. Then $q+r$ denotes the profile in $\mathbb{L}^{N_{3}}$, where $N_{3}=\left\{1,2, \ldots, n_{1}+n_{2}\right\}$ defined for each agent $i \in N_{3}$ by

$$
(q+r)(i)=\left\{\begin{array}{cl}
q(i) & \text { if } \quad i \in\left\{1,2, \ldots, n_{1}\right\} \\
r\left(i-n_{1}\right) & \text { if } i \in\left\{n_{1}+1, n_{1}+2, \ldots, n_{1}+n_{2}\right\}
\end{array} .\right.
$$

The order in this "addition" matters as in general $q+r \neq r+q$. For a sequence of profiles, say $q^{1}$ up to $q^{k}$, the profile $q^{1}+q^{2}+\cdots+q^{k}$ is denoted by $\sum_{t=1}^{k} q^{t}$.

\footnotetext{
5 We take linear orders to be irreflexive without theoretical consequences in our approach. It has however notational advantage here as now linear orders are partial tournaments.
} 
Let $\mathbb{1}$ denote the set of all partial tournaments on $A$, i.e., asymmetric binary relations on $A$. In the sequel we shall omit the term partial. For a subset $V$ of $\mathbb{T}$ let $V_{a b}$ $=\{T \in V: a b \in T\}$ denote the subset of partial tournaments in $V$ containing pair $a b$. Note that $\mathbb{L} \subseteq \mathbb{T}$. So, $\mathbb{L}_{a b}$ is the set of all linear orders ordering $a$ above $b$. A tournament $T$ is called acyclic if there is a linear order $R$ such that $T \subseteq R$. Let A denote the set of all acyclic tournaments.

Let $p$ be a preference profile and $a$ and $b$ be distinct alternatives. Then $\bar{p}_{a b}=|\{i: a b \in p(i)\}|$ denotes the number of agents ordering $a$ above $b$ at $p$. The (pairwise majority) tournament associated to profile $p$ is defined as follows: $T_{p}=\left\{a b \in A \times A: \bar{p}_{a b}>\bar{p}_{b a}\right\}$. That is, pair of alternatives $a b$ is in the tournament $T_{p}$, whenever the number of agents ordering $a$ above $b$ is strictly greater than the number of agents ordering $b$ above $a$.

The Kemeny distance between $T$ and $T^{\prime}$ is defined by $\delta\left(T, T^{\prime}\right)=\left|T \backslash T^{\prime}\right|+\left|T^{\prime} \backslash T\right|$, i.e., the cardinality of the symmetric set difference of $T$ and $T^{\prime}$. The extension of the Kemeny distance between a linear order $R$ and a profile $p \in \mathbb{L}^{N}$ is defined by $\delta(p, R)=\sum_{i \in N} \delta(p(i), R)$

Finally, a preference correspondence or rule $\varphi$ assigns a non-empty subset of linear orders $\varphi(p)$ to every profile $p \in \mathbb{L}^{N}$ for every agent set $N$. A rule aggregates the preferences of the agents into a set of linear orders $\varphi(p)$, the collective outcome at profile $p$. In case the pairwise majority tournament, $T_{p}$, contains cycles every linear order in the outcome orders some pairs on this cycle in a reversed way. It therewith violates the corresponding pairwise majority of the profile. The Slater rule chooses those linear orders at which as few as possible of such majority violations are required. Formally, for any profile $p$ the Slater rule is defined by,

$$
\varphi^{S}(p)=\left\{R \in \mathbb{L}: \delta\left(T_{p}, R\right) \leq \delta\left(T_{p}, R^{\prime}\right) \text { for all } R^{\prime} \in \mathbb{L}\right\} .
$$

\subsection{Elementary changes}

Next we define the notion of an elementary change in tournaments, linear orders and profiles. An elementary change is essentially "the smallest change possible" in a domain. This concept has slightly differing formulations in tournaments, linear orders, and profiles.

Let $a$ and $b$ be two distinct alternatives in $A$. We say that the ordered pair $\left(T, T^{\prime}\right)$ forms an elementary change (in $\mathbb{T}$ ) from $a b$ to $b a$ if either (i) $b a \in T^{\prime}$ and $T=T^{\prime} \backslash\{b a\}$ or (ii) $a b \in T$ and $T^{\prime}=T \backslash\{a b\}$. Note that $\left(T, T^{\prime}\right)$ forms an elementary change in $\mathbb{T}$ precisely when $\delta\left(T, T^{\prime}\right)=1$. For two linear orders $\left(R, R^{\prime}\right)$ we say $\left(R, R^{\prime}\right)$ forms an elementary change (in $\mathbb{L}$ ) from $a b$ to $b a$ if $R \backslash\{a b\}=R^{\prime} \backslash\{b a\}$. That is, $a$ is consecutively ordered above $b$ at $R$ and $b$ is so above $a$ at $R^{\prime}$, where in going from $R$ to $R^{\prime}$ only the positions of $a$ and $b$ are swapped. Note that $\delta\left(R, R^{\prime}\right)=2$ which is the minimum value for distinct linear orders. Finally, we define the notion of elementary change for two profiles. For some set of agents $N$ let $p$ and $q$ be two profiles in $\mathbb{L}^{N}$. We say that the ordered pair of profiles $(p, q)$ forms an elementary change (in $\mathbb{L}^{N}$ ) from $a b$ to $b a$, whenever for some agent $i \in N$ the pair $(p(i), q(i))$ forms 
an elementary change in $\mathbb{L}$ from $a b$ to $b a$, and $p(j)=q(j)$ for all $j \in N \backslash\{i\}$. So, elementary changes at profiles occur precisely when one agent swaps the ranking of two consecutively ordered alternatives and all other agents do not change their preferences. It is the "slightest" possible change between distinct profiles.

\subsection{Some fundamental profiles}

McGarvey (1953) introduced the following two agents profiles, denoted by $r^{a b, R}$, as building blocks for a given tournament, say $T$. Such a profile $r^{a b, R}$ is determined by two distinct alternatives $a$ and $b$ and a linear order $R$ such that $a$ is consecutively ordered above $b$ at $R$. Further, $r^{a b, R}(1)=R$ and $r^{a b, R}(2)$ is the linear order such that $r^{a b, R}(2)=(-R \backslash\{b a\}) \cup\{a b\}$. Note also that the pair of linear orders, $\left(-R, r^{a b, R}(2)\right)$ forms an elementary change from $b a$ to $a b$. We call the profile $r^{a b, R}$ an almost maximal conflict, as agent 1 and 2 only agree on one pair $a b$. An example for such a profile could be as follows: $r^{a b, R}(1)=a b c_{1} c_{2} \ldots c_{m-2}$ and $r^{a b, R}(2)=c_{m-2} \ldots c_{2} c_{1} a b$.

Note that for the almost maximal conflict profile $r^{a b, R}$, the associated tournament is $T_{r a b, R}=\{a b\}$. In case the choice of $R$ is not important we may write $r^{a b}$ instead of $r^{a b, R}$. Finally, let $T=\left\{x_{1} y_{1}, x_{2} y_{2}, \ldots, x_{k} y_{k}\right\}$ be an arbitrary tournament in $\mathbb{T}$. Then, we can associate a profile $r^{T}$ defined by $r^{T}=\sum_{x y \in T} r^{x y}$ to $T$ where $T$ is the associated tournament to $r^{T}$. Note that by McGarvey $(1953), T_{r^{T}}=T$.

\section{The characterizing conditions}

To characterize the Slater rule we introduce the following five conditions for a preference rule $\varphi$.

- Unbiased: $\varphi$ is unbiased (at uniform profiles) if for all agent sets $N$, and all uniform profiles $p \in \mathbb{L}^{N}$,

$$
\varphi(p)=\mathbb{L} .
$$

The condition imposes that at profiles, where all linear orders in $\mathbb{L}$ are reported the same number of times, the alternatives are treated equally. It can therewith be seen as a limited neutrality requirement which is satisfied by many rules.

- Monotone: $\varphi$ is (update) monotone if for all pairs of profiles $(p, q)$ forming an elementary change from $a b$ to $b a$, with $\varphi(p)_{b a} \neq \emptyset$,

$$
\varphi(p)_{b a} \subseteq \varphi(q) \subseteq \varphi(p) .
$$

In words, monotonicity requires that if an agent swaps a pair of adjacent ordered alternatives, here from $a b$ to $b a$, towards an outcome preference, then at this updated profile the outcome is a subset of the old outcome containing this specific preference. Note that this swapping is an update towards all the preferences at which $b$ is ordered above $a$. Therefore all preferences in $\varphi(p)_{b a}$ are in $\varphi(q)$. Update monotonicity is introduced by Can and Storcken (2013) as one of the characterizing conditions of the Kemeny rule. In Appendix , we relate update monotonicity to a well-known monotonicity condition for tournament correspondences. 
- Tournamental: $\varphi$ is tournamental if for all profiles $p$ and $q$, with $T_{p}=T_{q}$,

$$
\varphi(p)=\varphi(q) .
$$

Note that in this definition $p$ and $q$ need not stem from the same agent sets. It is clear that the condition of being tournamental imposes the rule to be "pairwise". But the Slater rule is not the only tournamental rule as explained in the introduction. Note that being tournamental imposes anonymity and replication invariance on the rule. The latter condition requires that the outcome of the rule does not change, in case the profile is expanded by replicating all agents' preferences the same number of times.

- Tie-breaking: $\varphi$ is tie-breaking if for all pairs of profiles $(p, q)$ forming an elementary change from $a b$ to $b a$ in $\mathbb{L}^{N}$, with $\bar{p}_{a b}=\bar{p}_{b a}$,

$$
\text { if } \varphi(p)_{b a} \neq \emptyset \text {, then } \varphi(q)_{a b}=\emptyset \text {. }
$$

In words, consider a profile $p$ in which a pairwise tie between $a$ and $b$ occurs. That is the number of agents preferring $a$ to $b$ at $p$ equals the number of agents preferring $b$ to $a$ at $p: \bar{p}_{a b}=\bar{p}_{b a}$. Suppose an agent who orders $a$ above $b$ consecutively in her preference (only) swaps those alternatives to get a new profile $q$. In these cases the condition requires that if (at the initial outcome) there was already a ranking that put $b$ above $a$, then after the switching to a strict pairwise majority of $b$ over $a$ there are no outcomes (at $q$ ) putting $a$ above $b$. Situations such that $\varphi(p)_{a b} \neq \emptyset$ and $\varphi(p)_{b a} \neq \emptyset$ are interpreted as a "tie" between $a$ and $b$ in the outcome $\varphi(p)$.

Remark 1 shows that for the Slater rule the "equal votes situation" is a necessary premise in the tie-breaking condition. That is, the Slater rule does not satisfy a similar tie-breaking condition applicable to a wider class of elementary changes, in particular at those where there are no equal votes.

Remark 1 Let $A=\{a, b, c\}$ be a set of three alternatives and $N=\{1,2, \ldots, 12\}$ be the set of agents. Consider profile $p \in \mathbb{L}^{N}$ defined as follows:

$$
\begin{aligned}
& p(i)=a b c \text { for } 1 \leq i \leq 4, \\
& p(i)=b c a \text { for } 5 \leq i \leq 8, \\
& p(i)=c a b \text { for } 9 \leq i \leq 12 .
\end{aligned}
$$

Further, consider profile $q \in \mathbb{L}^{N}$, such that $q(i)=p(i)$ for all $i \in\{1,2,3, \ldots, 11\}$ and let $q(12)=c b a$. Now $(p, q)$ forms an elementary change from $a b$ to $b a$. Yet $T_{p}=T_{q}=\{a b, b c, c a\}$ and the Slater rule assigns $\{a b c, b c a, c a b\}$ to both of these profiles. So, at both of the outcomes there is a tie between $a$ and $b$. Obviously, the equal votes requirement is violated at $p$ and $q$ and $\left(T_{p}, T_{q}\right)$ does not form an elementary change. This clarifies that the equal votes requirement is essential in the definition of tie-breaking.

- Gradual: $\varphi$ is gradual if for all pairs of profiles $(p, q)$ forming an elementary change from $a b$ to $b a$ in $\mathbb{L}^{N}$, with $\bar{p}_{a b}=\bar{p}_{b a}$, 


$$
\text { if } \varphi(q)_{a b}=\emptyset \text {, then } \varphi(p)_{b a} \neq \emptyset \text {. }
$$

In words, consider a profile $p$ in which a pairwise tie between $a$ and $b$ occurs. Suppose an agent who orders $a$ above $b$ consecutively in her preference (only) swaps those alternatives to get a new profile $q$. In these cases the condition requires that if at the new profile $q$ all outcomes put $b$ above $a$, then there exist at least some outcome at the old profile $p$ that put $b$ above $a$.

The following lemma shows that the Slater rule satisfies all the aforementioned conditions.

Lemma 1 The Slater rule is unbiased, tournamental, monotone, tie-breaking, and gradual.

Proof In the following we show that the Slater rule satisfies each of these conditions. Unbiased: As at a uniform profile the corresponding pairwise majority tournament is empty, it is evident that the Slater rule assigns the set of all linear orders to such a profile. Hence, the Slater rule is unbiased.

Tournamental: It follows from the definition that the Slater rule is tournamental.

Monotone: To show that the Slater rule satisfies monotonicity, we have to show that for all pairs of profiles $(p, q)$ forming an elementary change from $a b$ to $b a$, with $\varphi^{S}(p)_{b a} \neq \emptyset$, we have $\varphi^{S}(p)_{b a} \subseteq \varphi^{S}(q) \subseteq \varphi^{S}(p)$. Let $(p, q)$ be as such. In case $T_{p}=T_{q}$, as $\varphi^{S}$ is tournamental, then $\varphi^{S}(p)=\varphi^{S}(q)$ which results in monotonicity for this case. Therefore, let $T_{p} \neq T_{q}$. Take $R \in \varphi^{S}(p)_{b a}$. It suffices to show (i) $R \in \varphi^{S}(q)$ and (ii) $\varphi^{S}(q) \subseteq \varphi^{S}(p)$. Since $(p, q)$ forms an elementary change from $a b$ to $b a, T_{p} \backslash\{a b\}=T_{q} \backslash\{b a\}, b a \notin T_{p}$, and $a b \notin T_{p}$. So, $T_{p} \backslash R=\left(T_{q} \backslash R\right) \cup\left(T_{p} \backslash T_{q}\right)$ and $R \backslash T_{p}=\left(R \backslash T_{q}\right) \cup\left(T_{q} \backslash T_{p}\right)$. Hence,

$$
\delta\left(T_{p}, R\right)=\delta\left(T_{p}, T_{q}\right)+\delta\left(T_{q}, R\right) .
$$

(i) To the contrary assume $R \notin \varphi^{S}(q)$. It is sufficient to deduce a contradiction. As $R \notin \varphi^{S}(q)$ there is a linear order $R^{\prime}$ such that $\delta\left(T_{q}, R^{\prime}\right)<\delta\left(T_{q}, R\right)$. Adding $\delta\left(T_{p}, T_{q}\right)$ to both sides yields $\delta\left(T_{p}, T_{q}\right)+\delta\left(T_{q}, R^{\prime}\right)<\delta\left(T_{p}, T_{q}\right)+\delta\left(T_{q}, R\right)$. Applying Eq. (1) to the right hand side and triangle inequality to the left hand side of the latter inequality yields $\delta\left(T_{p}, R^{\prime}\right)<\delta\left(T_{p}, R\right)$. As we assumed $R \in \varphi^{S}(p)$, and therewith $\delta\left(T_{p}, R\right) \leq \delta\left(T_{p}, R^{\prime}\right)$, we have a contradiction. So, $R \in \varphi^{S}(q)$.

(ii) To show the inclusion $\varphi^{S}(q) \subseteq \varphi^{S}(p)$, take any $R^{\prime \prime} \in \varphi^{S}(q)$. It is sufficient to prove that $R^{\prime \prime} \in \varphi^{S}(p)$. Since $R \in \varphi^{S}(p)_{b a}$ (and therefore $R \in \varphi^{S}(p)$ ), it is sufficient to prove that $\delta\left(T_{p}, R^{\prime \prime}\right) \leq \delta\left(T_{p}, R\right)$. By triangle inequality of $\delta$ we have that $\delta\left(T_{p}, R^{\prime \prime}\right) \leq \delta\left(T_{p}, T_{q}\right)+\delta\left(T_{q}, R^{\prime \prime}\right)$. As $R^{\prime \prime} \in \varphi^{S}(q)$, it follows that $\delta\left(T_{q}, R^{\prime \prime}\right) \leq \delta\left(T_{q}, R\right)$ for any $R \in \varphi^{S}(p)_{b a}$. This together with the previous inequality yields $\delta\left(T_{p}, R^{\prime \prime}\right) \leq \delta\left(T_{p}, T_{q}\right)+\delta\left(T_{q}, R\right)$. Applying Eq. (1) on the right hand side of the latter weak inequality yields $\delta\left(T_{p}, R^{\prime \prime}\right) \leq \delta\left(T_{p}, R\right)$ for 
any $R \in \varphi^{S}(p)_{b a}$ and thus also for any $R \in \varphi^{S}(p)$ since $R \in \varphi^{S}(p)_{b a}$ implies $\delta\left(T_{p}, R\right)=\delta\left(T_{p}, R^{\prime \prime \prime}\right)$ for any $R^{\prime \prime \prime} \in \varphi^{S}(p)$. Therefore, $R^{\prime \prime} \in \varphi^{S}(p)$ which proves the inclusion $\varphi^{S}(q) \subseteq \varphi^{S}(p)$.

Tie-breaking: To show that the Slater rule satisfies tie-breaking we have to show that $\varphi^{S}(q)_{a b}=\emptyset$ for arbitrary pairs of profiles $(p, q)$ forming an elementary change in $\mathbb{L}^{N}$ from $a b$ to $b a$, with $\bar{p}_{a b}=\bar{p}_{b a}$ and $\varphi^{S}(p)_{b a} \neq \emptyset$. To the contrary assume $\varphi^{S}(q)_{a b} \neq \emptyset$. We deduce a contradiction. Since $(p, q)$ forms an elementary change from $a b$ to $b a$ and $\varphi^{S}(p)_{b a} \neq \emptyset$ monotonicity implies $\left(\varphi^{S}(p)_{b a} \subseteq\right) \varphi^{S}(q) \subseteq \varphi^{S}(p)$. Since $(q, p)$ forms an elementary change from $b a$ to $a b$ and $\varphi^{S}(q)_{a b} \neq \emptyset$ monotonicity implies $\left(\varphi^{S}(q)_{a b} \subseteq\right) \varphi^{S}(p) \subseteq \varphi^{S}(q)$. Therefore, $\varphi^{S}(p)=\varphi^{S}(q)$. As we have $\varphi^{S}(p)_{b a} \neq \emptyset$ let $R \in \varphi^{S}(p)=\varphi^{S}(q)$ with $b a \in R$. As we assumed to the contrary that $\varphi^{S}(q)_{a b} \neq \emptyset$ let $R^{\prime} \in \varphi^{S}(q)=\varphi^{S}(p)$ with $a b \in R^{\prime}$. As both $R$ and $R^{\prime}$ are in both $\varphi^{S}(q)$ and $\varphi^{S}(p)$ by the definition of the Slater rule we have

$$
\begin{aligned}
& \delta\left(T_{p}, R\right)=\delta\left(T_{p}, R^{\prime}\right), \\
& \delta\left(T_{q}, R\right)=\delta\left(T_{q}, R^{\prime}\right) .
\end{aligned}
$$

Similarly to (1) we have

$$
\begin{gathered}
\delta\left(T_{p}, R\right)=\delta\left(T_{p}, T_{q}\right)+\delta\left(T_{q}, R\right), \\
\delta\left(T_{q}, R^{\prime}\right)=\delta\left(T_{q}, T_{p}\right)+\delta\left(T_{p}, R^{\prime}\right) .
\end{gathered}
$$

Substituting Eq. (3) into Eq. (4) implies $\delta\left(T_{p}, R\right)=\delta\left(T_{p}, T_{q}\right)+\delta\left(T_{q}, R^{\prime}\right)$. Substituting Eq. (5) into this latter equation results in $\delta\left(T_{p}, R\right)=\delta\left(T_{p}, T_{q}\right)+\delta\left(T_{q}, T_{p}\right)+\delta\left(T_{p}, R^{\prime}\right)$. This together with Eq. (2) implies the contradiction that $\delta\left(T_{p}, T_{q}\right)=0$.

Gradual: To show that the Slater rule is gradual we have to show that $\varphi^{S}(p)_{b a} \neq \varnothing$ for an arbitrary elementary change $(p, q)$ from $a b$ to $b a$, with $\bar{p}_{a b}=\bar{p}_{b a}$ and $\varphi^{S}(q)_{a b}=\emptyset$. Let $p$ and $q$ be such profiles. Consider $T_{p}$ and $T_{q}$. As $\varphi^{S}(q)_{a b}=\emptyset$, it follows that $\varphi^{S}(q)_{b a}=\varphi^{S}(q) \neq \emptyset$. If $T_{p}=T_{q}$, then as the Slater rule is tournamental $\varphi^{S}(p)=\varphi^{S}(q)$. But $\varphi^{S}(q)_{b a}=\varphi^{S}(q)$. Hence, $\varphi^{S}(p)_{b a} \neq \emptyset$.

Now suppose that $T_{p} \neq T_{q}$. As $(p, q)$ is an elementary change from $a b$ to $b a$, with $\bar{p}_{a b}=\bar{p}_{b a}$, we have that $T_{p}=T_{q} \backslash\{b a\}$ and $b a \in T_{q}$. To the contrary assume $\varphi^{S}(p)_{b a}=\emptyset$. We deduce a contradiction. As $\varphi^{S}(q)_{a b}=\emptyset$ and $\varphi^{S}(p)_{b a}=\emptyset$ we have $\varphi^{S}(q) \subseteq \mathbb{L}_{b a}$ and $\varphi^{S}(p) \subseteq \mathbb{L}_{a b}$. Suppose that $R \in \varphi^{S}(p)$ and $R^{\prime} \in \varphi^{S}(q)$. First we prove that $\delta\left(R, T_{p}\right)=\delta\left(R^{\prime}, T_{q}\right)$. Similar to (1) we have

$$
\delta\left(T_{p}, R^{\prime}\right)=\delta\left(T_{p}, T_{q}\right)+\delta\left(T_{q}, R^{\prime}\right)=1+\delta\left(T_{q}, R^{\prime}\right) .
$$

Because of $R^{\prime} \notin \varphi^{S}(p)$, we have $\delta\left(T_{p}, R\right)<\delta\left(T_{p}, R^{\prime}\right)$. Hence, $1+\delta\left(T_{p}, R\right) \leq \delta\left(T_{p}, R^{\prime}\right)$. Combining this weak inequality and Eq. (6), yields $\delta\left(T_{p}, R\right) \leq \delta\left(T_{q}, R^{\prime}\right)$. The reverse weak inequality, $\delta\left(T_{q}, R^{\prime}\right) \leq \delta\left(T_{p}, R\right)$, follows similarly by interchanging the role of $T_{p}$ and $T_{q}$ and that of $R$ and $R^{\prime}$. So, we may conclude that $\delta\left(R, T_{p}\right)=\delta\left(R^{\prime}, T_{q}\right)$. 
Now we have a path of elementary changes in $\mathbb{T}$ from $R$ to $T_{p}$ of length $\delta\left(R, T_{p}\right)$ and a path of elementary changes in $\mathbb{T}$ from $T_{q}$ to $R^{\prime}$ of length $\delta\left(R^{\prime}, T_{q}\right)=\delta\left(R, T_{p}\right)$. As $\left(T_{p}, T_{q}\right)$ forms an elementary change, all in all there is a path of elementary changes in $\mathbb{T}$ from $R$ to $R^{\prime}$ of length $2 \times \delta\left(R, T_{p}\right)+\delta\left(T_{p}, T_{q}\right)=2 \times \delta\left(R, T_{p}\right)+1$. This is a path of odd length. But $R$ and $R^{\prime}$ are linear orders and therefore complete. Between complete tournaments every path of elementary changes in $\mathbb{T}$ has even length ${ }^{6}$. This contradiction completes the proof.

\section{Tournament correspondences}

A tournament (preference) correspondence $\Phi$ is a function which assigns to every tournament $T \in \mathbb{T}$ a non-empty subset of $\mathbb{L}$. To a tournamental rule $\varphi$ we associate a tournament (preference) correspondence $\Phi^{\varphi}$ which assigns to any $T \in \mathbb{T}$ a subset of $\mathbb{L}$ as follows:

$$
\Phi^{\varphi}(T)=\varphi(p), \text { where } p \text { is a profile such that } T=T_{p} .
$$

Note that by Sect. 2.3 for every tournament $T$, there is at least one profile $p$ such that $T=T_{p}$. So, $\Phi^{\varphi}$ is well-defined. As the Slater rule, $\varphi^{S}$, is tournamental we have a corresponding tournament correspondence $\Phi^{\varphi^{S}}$. For simplicity, in what follows we shall denote it by $\Phi^{S}$ instead.

Next we introduce conditions on tournament correspondences. Essentially, these are adaptations of the conditions discussed in Sect. 3. To distinguish these conditions on tournament correspondences from those on preference rules, we add a letter " $T$ " at the front. Let $\Phi$ be a tournament correspondence.

- T-unbiased: $\Phi$ is T-unbiased if $\Phi(\emptyset)=\mathbb{L}$.

- T-monotone: $\Phi$ is T-monotone if for all tournament pairs $\left(T, T^{\prime}\right)$ forming an elementary change in $\mathbb{T}$ from $a b$ to $b a$, with $\Phi(T)_{b a} \neq \emptyset$,

$$
\Phi(T)_{b a} \subseteq \Phi\left(T^{\prime}\right) \subseteq \Phi(T) .
$$

- T-tie-breaking: $\Phi$ is T-tie-breaking if for all tournament pairs $\left(T, T^{\prime}\right)$ forming an elementary change in $\mathbb{1}$ from $a b$ to $b a$,

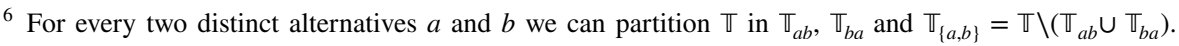
Note that complete tournaments are in $\mathbb{T}_{a b} \cup \mathbb{\mathbb { T }}_{b a}$ and that $\mathbb{T}_{\{a, b\}}$ consists of the incomplete tournaments at which $a$ and $b$ are incomparable. Every elementary change from $a b$ to $b a$ or from $b a$ to $a b$ is now a connecting relation (edge) between an element in $\mathbb{T}_{a b} \cup \mathbb{T}_{b a}$ and one in $\mathbb{T}_{\{a, b\}}$. Further, an elementary change from $x y$ to $y x$ does not effect the preference between $a$ and $b$ in case $\{x, y\} \neq\{a, b\}$. Call an elementary change between $a$ and $b$ if it is either from $a b$ to $b a$ or from $b a$ to $a b$. So, a path of elementary changes with an odd number of changes between $a$ and $b$ starting in $\mathbb{T}_{a b} \cup \mathbb{\mathbb { T }}_{b a}$ ends in $\mathbb{\mathbb { T }}_{\{a, b\}}$. As $\mathbb{\mathbb { T }}_{\{a, b\}}$ consists of incomplete tournaments and both $R$ an $R^{\prime}$ are complete it follows that on every path of elementary changes from $R$ to $R^{\prime}$ there is an even number of elementary changes between $a$ and $b$. As this holds for every two distinct alternatives it follows that such a path is of even length.
} 


$$
\text { if } \Phi(T)_{b a} \neq \emptyset \text {, then } \Phi\left(T^{\prime}\right)_{a b}=\emptyset \text {. }
$$

- T-gradual: $\Phi$ is T-gradual if for all tournament pairs $\left(T, T^{\prime}\right)$ forming an elementary change in $\mathbb{T}$ from $a b$ to $b a$,

$$
\text { if } \Phi\left(T^{\prime}\right)_{a b}=\emptyset \text {, then } \Phi(T)_{b a} \neq \emptyset .
$$

The following lemma states that the tournament correspondence $\Phi^{\varphi}$ inherits these "T" properties from the tournamental preference rule $\varphi$.

Lemma 2 (Inheritance Lemma) Let $\varphi$ be an unbiased, monotone, tournamental, tiebreaking and gradual rule. Then the associated tournament correspondence $\Phi^{\varphi}$ is T-unbiased, T-monotone, T-tie-breaking, and T-gradual.

Proof $\Phi^{\varphi}$ is obviously T-unbiased.

Next we show that the tournament correspondence $\Phi^{\varphi}$ inherits the other three conditions from $\varphi$. To that end, consider any tournament pair $\left(T, T^{\prime}\right)$ forming an elementary change from $a b$ to $b a$ with distinct alternatives $a$ and $b$. Then either of the following two holds

1. $a b \notin T, b a \notin T$, and $T^{\prime}=T \cup\{b a\}$,

2. $a b \notin T^{\prime}, b a \notin T^{\prime}$ and $T=T^{\prime} \cup\{a b\}$

That is, in the first case, $a$ and $b$ are incomparable in $T$, while $T^{\prime}$ puts $b$ above $a$. Correspondingly in the second case $a$ and $b$ are incomparable in $T^{\prime}$, while $T$ puts $a$ above $b$. Using the building blocks of Sect. 2.3, we build a preference profile $p$ (and respectively $q$ ) for which $T$ (and respectively $T^{\prime}$ ) is the associated tournament.

In case (1), let $r^{T}=\sum_{x y \in T} r^{x y}$. Take any linear order $R^{\prime}$ at which $b$ is consecutively ordered above $a$ and construct $q=r^{T}+r^{b a, R^{\prime}}$. Further in this case, let $p=r^{T}+o^{R^{\prime}}$, where $o^{R^{\prime}}=\left(R^{\prime},-R^{\prime}\right)$ is a two agent profile. Note that $(p, q)$ forms an elementary change from $a b$ to $b a$, with $\bar{p}_{a b}=\bar{p}_{b a}$ and $T_{p}=T$ and $T_{q}=T^{\prime}$.

In case (2), let $r^{T^{\prime}}=\sum_{x y \in T^{\prime}} r^{x y}$. Take any linear order $R$ at which $a$ is consecutively ordered above $b$ and construct $p=r^{T^{\prime}}+r^{a b, R}$. Further in this case, let $q=r^{T}+o^{R}$, where $o^{R}=(R,-R)$ is a two agent profile. Note that $(p, q)$ forms an elementary change from $a b$ to $b a$, with $\bar{q}_{a b}=\bar{q}_{b a}$ and $T_{p}=T$ and $T_{q}=T^{\prime}$.

$\Phi^{\varphi}$ is T-monotone: Consider the aforementioned $\left(T, T^{\prime}\right)$. In addition, assume $\Phi^{\varphi}(T)_{b a} \neq \emptyset$. It is sufficient to show the following two inclusions $\Phi^{\varphi}(T)_{b a} \subseteq$ $\Phi^{\varphi}\left(T^{\prime}\right) \subseteq \Phi^{\varphi}(T)$. Because $\Phi^{\varphi}(T)_{b a} \neq \emptyset$, and $T$ is the associated tournament of $p$, it follows by the definition of $\Phi^{\varphi}$ that $\varphi(p)_{b a}=\Phi^{\varphi}(T)_{b a} \neq \emptyset$. Monotonicity of $\varphi$ implies $\varphi(p)_{b a} \subseteq \varphi(q) \subseteq \varphi(p)$. Finally, as $T^{\prime}$ is the associated tournament of $q$, the definition of $\Phi^{\varphi}$ now yields $\Phi^{\varphi}(T)_{b a} \subseteq \Phi^{\varphi}\left(T^{\prime}\right) \subseteq \Phi^{\varphi}(T)$.

$\Phi^{\varphi}$ is T-tie-breaking: Consider the aforementioned pair $\left(T, T^{\prime}\right)$ and let $\Phi^{\varphi}(T)_{b a} \neq \emptyset$. It is sufficient to show that $\Phi^{\varphi}\left(T^{\prime}\right)_{a b}=\emptyset$.

If case 1 applies to $\left(T, T^{\prime}\right)$, then we have equal votes at $p$. As $\Phi^{\varphi}(T)_{b a} \neq \emptyset$, then $\varphi(p)_{b a} \neq \emptyset$ and tie-breaking of $\varphi$ imply $\varphi(q)_{a b}=\emptyset$. Hence, $\Phi^{\varphi}\left(T^{\prime}\right)_{a b}=\emptyset$. 
If case 2 applies to $\left(T, T^{\prime}\right)$, then we have equal votes at $q$. If in this case $\Phi^{\varphi}\left(T^{\prime}\right)_{a b} \neq \emptyset$, then $\varphi(q)_{a b} \neq \emptyset$. Tie-breaking of $\varphi$ would imply $\varphi(p)_{b a}=\emptyset$, and therefore $\Phi^{\varphi}(T)_{b a}=\emptyset$, which is a contradiction. Hence, $\Phi^{\varphi}\left(T^{\prime}\right)_{a b}=\emptyset$.

$\Phi^{\varphi}$ is T-gradual: Consider the aforementioned pair $\left(T, T^{\prime}\right)$. Let $\Phi^{\varphi}\left(T^{\prime}\right)_{a b}=\emptyset$. It is sufficient to show that $\Phi^{\varphi}(T)_{b a} \neq \varnothing$.

If case 1 applies to $\left(T, T^{\prime}\right)$, then we have equal votes at $p$. Now $\Phi^{\varphi}\left(T^{\prime}\right)_{a b}=\emptyset$ implies $\varphi(q)_{a b}=\emptyset$. So, graduality of $\varphi$ implies $\varphi(p)_{b a} \neq \emptyset$. Hence, $\Phi^{\varphi}(T)_{b a} \neq \emptyset$.

If case 2 applies to $\left(T, T^{\prime}\right)$, then we have equal votes at $q$. If in this case $\Phi^{\varphi}(T)_{b a}=\emptyset$, then $\varphi(p)_{b a}=\emptyset$. Graduality of $\varphi$ would imply that $\varphi(q)_{a b} \neq \emptyset$, and therewith the contradiction $\Phi^{\varphi}\left(T^{\prime}\right)_{a b} \neq \emptyset$. Hence, $\Phi^{\varphi}(T)_{b a} \neq \emptyset$.

Next we discuss two elementary results. The following result shows that combining T-monotonicity and T-tie-breaking yields a stronger monotonicity condition.

Lemma 3 Let $\Phi$ be a T-monotone and T-tie-breaking tournament correspondence. Let tournament pair $\left(T, T^{\prime}\right)$ forms an elementary change from ab to ba. Let $\Phi(T)_{b a} \neq \emptyset$. Then $\Phi\left(T^{\prime}\right)=\Phi(T)_{b a}$.

Proof Consider a tournament correspondence $\Phi$, tournaments $T$ and $T^{\prime}$, and alternatives $a$ and $b$ as in the formulation of the Lemma. Because $\Phi(T)_{b a} \neq \emptyset$, monotonicity and tie-breaking imply $\Phi(T)_{b a} \subseteq \Phi\left(T^{\prime}\right) \subseteq \Phi(T)$ and $\Phi\left(T^{\prime}\right)_{a b}=\emptyset$. So, $\Phi(T)_{b a} \subseteq \Phi\left(T^{\prime}\right), \Phi\left(T^{\prime}\right) \subseteq \Phi(T)$, and $\Phi\left(T^{\prime}\right) \subseteq \mathbb{L}_{b a}$. Hence, $\Phi(T)_{b a} \subseteq \Phi\left(T^{\prime}\right) \subseteq \Phi(T)_{b a}$. So, $\Phi\left(T^{\prime}\right)=\Phi(T)_{b a}$.

The following result shows that at acyclic tournaments all T-unbiased, T-monotone, T-tie-breaking, and T-gradual tournament correspondences assign the set of complete linear extensions of these tournaments.

Lemma 4 Let $\Phi$ be a T-unbiased, T-monotone, T-tie-breaking, and T-gradual tournament correspondence. Let $T$ be an acyclic tournament. Then

$$
\Phi(T)=\bigcap_{a b \in T} \mathbb{L}_{a b}=\{R \in \mathbb{L}: T \subseteq R\} .
$$

Proof Let $T$ be an acyclic tournament. We show by induction on the size of $T$ that for such correspondences the outcome at such tournaments $T$, consists of all complete linear extensions of $T$, i.e., $\Phi(T)=\{R \in \mathbb{L}: T \subseteq R\}=\bigcap_{a b \in T} \mathbb{L}_{a b}$. As $\Phi(T)$ is T-unbiased, this holds for the empty tournament. Let $\left(T, T^{\prime}\right)$ be a pair of acyclic tournaments forming an elementary change from $x y$ to $y x$, such that $T^{\prime}=T \cup\{y x\}$. Furthermore, let $\Phi(T)=\{R \in \mathbb{L}: T \subseteq R\}=\bigcap_{a b \in T} \mathbb{L}_{a b}$. Because $T^{\prime}$ is acyclic there is a linear order $R^{\prime}$, such that $R^{\prime} \supseteq T^{\prime}$. So, $R^{\prime} \supseteq T^{a b \in T} \supseteq T$ and $y x \in T^{\prime} \subseteq R^{\prime}$. So, $R^{\prime} \in$ $\Phi(T)_{y x}$ by the assumptions on $\Phi(T)$. Now Lemma 3 implies $\Phi\left(T^{\prime}\right)=\Phi(T)_{y x}$. Hence, $\Phi\left(T^{\prime}\right)=\left\{R \in \mathbb{L}: T^{\prime} \subseteq R\right\}=\bigcap_{a b \in T^{\prime}} \mathbb{L}_{a b}$ and consists therewith of all linear orders extending $T^{\prime}$. All in all the above reasoning yields an inductive proof for the lemma. 


\section{Characterization}

Lemma 1 shows that the characterizing conditions of the Slater rule are necessary. Therefore, the proof of Theorem 1, dealing with the characterization of the Slater rule, is just on the sufficiency of these conditions.

Theorem 1 The Slater rule is the only rule that is simultaneously unbiased, monotone, tournamental, tie-breaking, and gradual.

Proof Lemma 1 shows that the Slater rule satisfies these properties. Let $\varphi$ be a rule that is unbiased, monotone, tournamental, tie-breaking, and gradual. We show that $\varphi$ and $\varphi^{S}$ are equal. Because both rules are tournamental it is sufficient to show that $\Phi^{\varphi}$ and $\Phi^{S}$ are equal. Lemma 2 implies that both these tournament correspondences are T-unbiased, T-monotone, T-tie-breaking, and T-gradual. It is therefore sufficient to show that any two T-unbiased, T-monotone, T-tie-breaking, and T-gradual tournament correspondences are equal. Let $\Phi^{1}$ and $\Phi^{2}$ be two such tournament correspondences. It is sufficient to prove for all $k \geq 0$ statement $\mathcal{P}(k)$ indicating

$$
\Phi^{1}(T)=\Phi^{2}(T) \text { for any tournament } T \in \mathbb{T} \text { with }|T| \leq k .
$$

where $|T|$ denotes the cardinality of the set $T$. This proof is by induction on $k$.

Basis $k=0$. In this case $T=\emptyset$. As $\Phi^{1}$ and $\Phi^{2}$ are T-unbiased $\Phi^{1}(\emptyset)=\mathbb{L}=\Phi^{2}(\emptyset)$. This completes the proof of $\mathcal{P}(0)$.

Induction Step. Assume $\mathcal{P}(k)$ holds. In order to prove $\mathcal{P}(k+1)$ let $T$ be a tournament with $|T|=k+1$. For reasons of symmetry it is sufficient to prove $\Phi^{1}(T) \subseteq \Phi^{2}(T)$. Take $R \in \Phi^{1}(T)$. It is sufficient to show that $R \in \Phi^{2}(T)$. We distinguish the following two cases: (i) $T \subseteq R$ and (ii) $T \nsubseteq R$.

Case (i) $T \subseteq R$. Let $T=\left\{x_{1} y_{1}, \ldots, x_{k} y_{k}\right\}$. By Lemma 4 we have $\Phi^{1}(T)=\mathbb{L}_{x_{1} y_{1}} \cap \mathbb{L}_{x_{2} y_{2}} \cap \cdots \cap \mathbb{L}_{x_{k} y_{k}}=\Phi^{2}(T)$. Hence, $R \in \Phi^{2}(T)$ which completes the analysis for this case.

Case (ii) $T \nsubseteq R$. If $T \nsubseteq R$ then there is a pair $a b \in T$ such that $b a \in R$. So, $R \in \Phi^{1}(T)_{b a}$. Let $T^{\prime}=T \backslash\{a b\}$. Then the tournament pair $\left(T, T^{\prime}\right)$ forms an elementary change from $a b$ to $b a$, and $\left|T^{\prime}\right|=|T|-1=k$. By T-monotonicity and T-tiebreaking of $\Phi^{1}$, Lemma 3 yields

$$
\Phi^{1}(T)_{b a}=\Phi^{1}\left(T^{\prime}\right) .
$$

As $\left|T^{\prime}\right|=|T|-1=k$, the induction hypothesis $\mathcal{P}(k)$ implies that

$$
\Phi^{1}\left(T^{\prime}\right)=\Phi^{2}\left(T^{\prime}\right) .
$$

Note that $\left(T^{\prime}, T\right)$ forms an elementary change from $b a$ to $a b$. In addition by Eqs. (7) and (8) we have $\Phi^{2}\left(T^{\prime}\right) \subseteq \mathbb{L}_{b a}$. Therefore, as $\Phi^{2}$ is T-gradual we have $\Phi^{2}(T)_{b a} \neq \emptyset$. As $\Phi^{2}$ is T-monotone and T-tie-breaking, Lemma 3 implies

$$
\Phi^{2}(T)_{b a}=\Phi^{2}\left(T^{\prime}\right) .
$$


Eqs. (7), (8), and (9) yield $\Phi^{1}(T)_{b a}=\Phi^{2}(T)_{b a}$. As $R \in \Phi^{1}(T)_{b a}$, we have $R \in \Phi^{2}(T)$. This proves $\Phi^{1}(T) \subseteq \Phi^{2}(T)$.

\section{Discussion}

Theorem 1 characterizes the Slater rule. Further, in Can et al. (2018) we show that the Kemeny rule can be characterized by a similar set of conditions along a similar line of arguments. Considering Lemmas 1 and 2 and in addition the proof of Theorem 1 Corollary 1 below follows as a kind of by-product. This Corollary is closer to the literature on pair comparisons as discussed by for instance Slater (1961) and Monsuur and Storcken (1997).

Corollary $1 \Phi^{S}$ is the only tournament correspondence which is simultaneously T-unbiased, T-monotone, T-tie-breaking, and T-gradual.

Although Remark 1 suggests that the tie-breaking condition cannot be weakened, others such as being gradual can. ${ }^{7}$ As the presented conditions are logically independent we chose these for presentational reasons. To show that the characterizing conditions in Theorem 1 are logically independent we define the following five rules at an arbitrary profile $p$ in $\llbracket^{N}$ as follows:

1. The constant $R$ rule: $\varphi^{R}(p)=\{R\}$,

2. The Kemeny(-Young) rule: $\varphi^{K}(p)=\left\{R \in \mathbb{L}: \kappa(R, p) \leq \kappa\left(R^{\prime}, p\right)\right.$ for all $\left.R^{\prime} \in \mathbb{L}\right\}$, where $\kappa(R, p)=\sum_{x y \in R} \bar{p}_{y x}$ (see Can and Storcken (2013); Young and Levenglick (1978)). ${ }^{8}$ This rule and the Slater rule these rules have sometimes been confused. ${ }^{9}$

3. The indecisive rule: $\varphi^{\mathbb{1}}(p)=\mathbb{L}$,

4. The Copeland rule: $\varphi^{C}(p)=\{R \in L: x y \in R$ for all $x, y \in A$, with Cscore $(x, p)>C \operatorname{cscore}(y, p)\}$, where Cscore $(a, p)=\left|\left\{b \in A: a b \in T_{p}\right\}\right|$,

5. The hierarchical rule: $\varphi^{>}(p)=\left\{R \in \mathbb{L}: \widehat{T}_{p} \subseteq R\right\}$, where among all acyclic tournaments contained in $T_{p}$ tournament $\widehat{T}_{p}$ is the best with respect to a given hierarchy, $>$, a linear order on the set of all acyclic tournaments $\mathbb{A}$ based on weights as

\footnotetext{
${ }_{7}$ For instance, it is possible to deduce the same results as presented here with only marginal changes based on a condition like $\varphi$ is gradual if for all pairs of profiles $(p, q)$ forming an elementary change from $a b$ to $b a$ in $\mathbb{L}^{N}$ there are non-negative integers $k$ such that

$$
\varphi(k \cdot p+q) \cap \varphi(p) \neq \emptyset .
$$

Meaning that if at such an elementary change the sets of outcomes $\varphi(p)$ and $\varphi(q)$ are disjoint then graduality imposes that for some (large) replica $k \cdot p$ of $p$ the influence of $q$ is limited. Meaning that the outcome at $k \cdot p+q$ has something in common with the outcome at $p$. This condition is related to Young's continuity condition for scoring rules. See Young (1975).

${ }^{8}$ This resembles the Fishburn's C2 condition for choice correspondences.

${ }^{9}$ See footnote 5 in Baigent and Klamler (2003), which attributes the clarification of the issue to Hannu Nurmi.
} 
Table 1 Logical independence of the conditions

\begin{tabular}{lccccc}
\hline Condition & $\varphi^{R}$ & $\varphi^{K}$ & $\varphi^{C}$ & $\varphi^{\complement}$ & $\varphi^{\succ}$ \\
\hline Unbiased & $-{ }^{1}$ & $+^{1}$ & $+^{1}$ & $+^{1}$ & $+^{1}$ \\
Tournamental & $+^{1}$ & $-{ }^{2}$ & $+^{1}$ & $+^{1}$ & $+^{1}$ \\
Monotone & $+^{1}$ & $+^{3}$ & $-{ }^{6}$ & $+^{1}$ & $+^{9}$ \\
Tie-breaking & $+^{1}$ & $+^{4}$ & $+^{7}$ & $-{ }^{1}$ & $+^{10}$ \\
Gradual & $+^{1}$ & $+^{5}$ & $+^{8}$ & $+^{1}$ & $-{ }^{11}$ \\
\hline
\end{tabular}

+: satisfied, -: unsatisfied

follows. Let $\omega$ be an injective function on the ordered pairs of distinct alternatives (in $A$ ) to the set of positive powers of 2 . For two acyclic tournaments $T^{1}$ and $T^{2}$ :

$$
T^{1}>T^{2} \text { if } \sum_{x y \in T^{1}} \omega(x y)>\sum_{x y \in T^{2}} \omega(x y)
$$

Note that because $\omega$ is injective and assigns powers of 2 , then $\widehat{T}_{p}$ is uniquely defined $^{10}$. Also note that, $\varphi^{\succ}(p)=\Phi^{S}\left(\widehat{T}_{p}\right)$.

Example 1 Let $A=\{a, b, c\}$. Let $\omega(b c)=2^{1}, \omega(a c)=2^{2}, \omega(a b)=2^{3}, \omega(b a)=2^{4}$, $\omega(c a)=2^{5}$, and $\omega(c b)=2^{6}$. Let $N=\{1, \ldots, 6\}$. Consider the following profiles:

$$
\begin{aligned}
& p=(a c b, a c b, c b a, c a b, b a c, b a c), \\
& q=(a c b, a c b, c b a, c b a, b a c, b a c) .
\end{aligned}
$$

Note that $T_{p}=\{a c, c b\}$. So, $\widehat{T}_{p}=T_{p}$ and $\varphi^{>}(p)=\{a c b\}$. As $T_{q}=\{a c, c b, b a\}$ the acyclic tournaments contained in $T_{q}$ are $T^{0}=\emptyset, T^{1}=\{a c\}, T^{2}=\{c b\}, T^{3}=\{b a\}$, $T^{4}=\{a c, c b\}, T^{5}=\{c b, b a\}$, and $T^{6}=\{b a, a c\}$. Denoting $\sum_{x y \in T} \omega(x y)$ by $\omega(T)$ yields: $\omega\left(T^{0}\right)=0, \omega\left(T^{1}\right)=4, \omega\left(T^{2}\right)=64, \omega\left(T^{3}\right)=16, \omega\left(T^{4}\right)=68, \omega\left(T^{5}\right)=80$, and $\omega\left(T^{6}\right)=20$. Therefore, $\widehat{T}_{q}=T^{5}$ and $\varphi^{>}(q)=\{c b a\}$.

Table 1 summarizes these results and shows this independence. In the following list of explanations, the list number corresponds to the superscripts in Table 1.

1. These are obvious or elementary.

2. To show that the Kemeny rule is not tournamental, consider the two profiles $p$ and $q$ on three alternatives with $p=(a b c, a b c, b c a, b c a, c a b, c a b, a b c)$ and $q=(a b c, a b c, b c a, b c a, c a b, c a b, b c a)$. It can be verified that $T_{p}=T_{q}=\{a b, b c, c a\}$. Yet $\varphi^{K}(p)=\{a b c\}$ and $\varphi^{K}(q)=\{b c a\}$.

\footnotetext{
${ }^{10}$ Instead of representing $>$ by an additive weight function $\omega$ as above one may choose $>$ such that
}

(a) $R^{1}>R^{2}$ for all $R^{1}, R^{2} \in \mathbb{A}$, with $R^{2} \subsetneq R^{1}$.

(b) $R^{1}>R^{2}$ for all $R^{1}, R^{2} \in \mathbb{A}$, with $R^{1} \backslash\{x y\}>R^{2} \backslash\{x y\}$ for some $x y \in R^{1} \cap R^{2}$.

Here $\widehat{T}_{p}$ being the best means that $\widehat{T}_{p}$ is that tournament in A contained in $T_{p}$ such that $\widehat{T}_{p}>T$, for all $T \in \mathbb{A} \backslash\left\{\widehat{T}_{p}\right\}$ and $T \subseteq T_{p}$. 
3. This monotonicity condition is one of the conditions by which the Kemeny rule is characterized in Can and Storcken (2013).

4. The Kemeny rule is tie-breaking. Recall that $\varphi^{K}(p)=\left\{R \in \mathbb{L}: \kappa(R, p) \leq \kappa\left(R^{\prime}, p\right)\right.$ for all $\left.R^{\prime} \in \mathbb{L}\right\}$. Now, let profile pair $(p, q)$ forms an elementary change from $a b$ to $b a$ and let $\bar{p}_{a b}=\bar{p}_{b a}$. So, the set of agents, say $N$, has even cardinality. As profile pair $(p, q)$ forms an elementary change from $a b$ to $b a$, and cardinality of $N$ is even, we have $\bar{q}_{x y}=\bar{p}_{x y}$ for all entries $x y$ not equal to $a b$ and not equal to $b a$, where $\bar{q}_{a b}=\bar{p}_{a b}-1$ and $\bar{q}_{b a}=\bar{p}_{b a}+1$. Let $R^{b a} \in \varphi^{K}(p)_{b a}$ and $R^{a b} \in \mathbb{L}_{a b}$. In order to prove that $\varphi^{K}$ is tie-breaking, it is sufficient to show that $R^{a b} \notin \varphi^{K}(q)$. As $\bar{q}_{a b}=\bar{p}_{a b}-1$ and $\bar{p}_{b a}+1=\bar{q}_{b a}$ and $R^{b a}$ is in $\varphi^{K}(p)$ we have $\kappa\left(R^{b a}, q\right)<\kappa\left(R^{b a}, p\right)$ $\leq \kappa\left(R^{a b}, p\right)<\kappa\left(R^{a b}, q\right)$. This means that $R^{a b} \notin \varphi^{K}(q)$.

5. The Kemeny rule is gradual. Let profile pair $(p, q)$ forms an elementary change from $a b$ to $b a$ and $\bar{p}_{a b}=\bar{p}_{b a}$. We are done if $\varphi^{K}(p)_{b a} \neq \emptyset$ or $\varphi^{K}(q)_{a b} \neq \emptyset$. To prove this, we show that the complementary case $\varphi^{K}(p)_{b a}=\emptyset$ and $\varphi^{K}(q)_{a b}=\emptyset$, leads to a contradiction. So, let $\varphi^{K}(q) \subseteq \mathbb{L}_{b a}$ and $\varphi^{K}(p) \subseteq \mathbb{L}_{a b}$. Let $R^{1} \in \varphi^{K}(p)$ and $R^{2} \in \varphi^{K}(q)$. So, $\sum_{x y \in R^{i} \cap(A \backslash\{a, b\})^{2}} \bar{p}_{y x}=\sum_{x y \in R^{i} \cap(A \backslash\{a, b\})^{2}} \bar{q}_{y x}$ for $i \in\{1,2\}$. We denote the values of these sums by $\alpha^{i}$. As $\bar{p}_{a b}=\bar{p}_{b a}$ we have $\sum_{x y \in R^{1} \cap\{a, b\}^{2}} \bar{p}_{y x}=\sum_{x y \in R^{2} \cap\{a, b\}^{2}} \bar{p}_{y x}$. We denote these sums by $\beta$. Further, we denote $\gamma^{i}=\sum_{x y \in R^{i} \cap\{a, b\}^{2}} \bar{q}_{y x}$ for $i \in\{1,2\}$. Because $\bar{q}_{a b}=\bar{p}_{a b}-1$ and $\bar{q}_{b a}=\bar{p}_{b a}+1$ we have $\left|\gamma^{1}-\gamma^{2}\right| \leq 2$. Now, $\kappa\left(R^{i}, p\right)=\alpha^{i}+\beta$ and $\kappa\left(R^{i}, q\right)=\alpha^{i}+\gamma^{i}$ for $i \in\{1,2\}$. As the number of agents is even $\left|\bar{r}_{x y}-\bar{r}_{y x}\right|$ is even for every profile $r \in \mathbb{L}^{N}$ and every two distinct alternatives $x$ and $y$. So, $\left|\kappa\left(R^{1}, p\right)-\kappa\left(R^{2}, p\right)\right|$ and $\left|\kappa\left(R^{1}, q\right)-\kappa\left(R^{2}, q\right)\right|$ are both even numbers. By the definition of $\varphi^{K}$ we have that $\kappa\left(R^{1}, p\right)<\kappa\left(R^{2}, p\right)$ and $\kappa\left(R^{2}, q\right)<\kappa\left(R^{1}, q\right)$. So, there are strictly positive integers $s$ and $t$ such that $\alpha^{1}+\beta+2 s=\kappa\left(R^{1}, p\right)+2 s=\kappa\left(R^{2}, p\right)=\alpha^{2}+\beta$ and $\alpha^{2}+\gamma^{2}+2 t=\kappa\left(R^{2}, q\right)+2 t=\kappa\left(R^{1}, q\right)=\alpha^{1}+\gamma^{1}$. Adding these two equalities yields $\alpha^{1}+\beta+2 s+\alpha^{2}+\gamma^{2}+2 t=\alpha^{2}+\beta+\alpha^{1}+\gamma^{1}$. Hence, $\gamma^{2}+2(s+t)=\gamma^{1}$. Because $s$ and $t$ are strictly positive this means that $\gamma^{1}$ and $\gamma^{2}$ differ by at least 4 . But this contradicts $\left|\gamma_{1}-\gamma_{2}\right| \leq 2$.

6. To show that the Copeland rule does not satisfy the monotonicity condition, consider the following two profiles $p$ and $q$ on three alternatives with $p=(b a c, a c b, c b a)$ and $q=(b a c, a c b, b c a)$. Note that $(p, q)$ forms an elementary change from $c b$ to $b c$. It can be verified that $T_{p}=\{b a, a c, c b\}$ and $T_{q}=\{b a, a c, b c\}$. So, Cscore $(a, p)=\operatorname{Cscore}(b, p)=\operatorname{Cscore}(c, p)=1$ and $\operatorname{Cscore}(b, q)=2, \operatorname{Cscore}(a, q)=1$ and $\operatorname{Cscore}(c, q)=0$. Hence, $\varphi^{C}(p)=\mathbb{L}$ and $b c a \in \varphi^{C}(p)$, however $b c a \notin \varphi^{C}(q)=\{b a c\}$.

7. The Copeland rule is tie-breaking. Let profile pair $(p, q)$ forms an elementary change from $a b$ to $b a$ and let $\bar{p}_{a b}=\bar{p}_{b a}$. Then tournament pair $\left(T_{p}, T_{q}\right)$ forms an elementary change from $a b$ to $b a$, such that $T_{p}=T_{q} \backslash\{b a\}$, and $b a \in T_{q}$. Note that all Cscores except that of $b$ are unchanged going from $p$ to $q$ and that of $b$ increases by 1 . It is sufficient to prove

$$
\text { if } \varphi^{C}(p)_{b a} \neq \emptyset \text { then } \varphi^{C}(q)_{a b}=\emptyset \text {. }
$$


As $\varphi^{C}(p)_{b a} \neq \emptyset$ then $\operatorname{Cscore}(b, p) \geq \operatorname{Cscore}(a, p)$. Given the scores changes going from $p$ to $q$ we have $\operatorname{Cscore}(b, q)>\operatorname{Cscore}(a, q)$. Since Cscore $(b, q)>\operatorname{Cscore}(a, q)$ then $\varphi^{C}(q)_{a b}=\emptyset$.

8. The Copeland rule is gradual. Let profile pair $(p, q)$ forms an elementary change from $a b$ to $b a$ and let $\bar{p}_{a b}=\bar{p}_{b a}$. Then tournament pair $\left(T_{p}, T_{q}\right)$ forms an elementary change from $a b$ to $b a$, such that $T_{p}=T_{q} \backslash\{b a\}$, and $b a \in T_{q}$. Note that all Cscores except that of $b$ are unchanged going from $p$ to $q$ and that of $b$ increases by 1 . It is sufficient to prove

$$
\varphi^{C}(q)_{a b}=\emptyset \text { then } \varphi^{C}(p)_{b a} \neq \emptyset .
$$

As $\varphi^{C}(q)_{a b}=\emptyset$ then $\operatorname{Cscore}(b, q)>\operatorname{Cscore}(a, q)$. Given the scores changes going from $p$ to $q$ we have $C \operatorname{score}(b, p) \geq C \operatorname{Cscore}(a, p)$. Since, Cscore $(b, p) \geq C \operatorname{Cscore}(a, p)$ then $\varphi^{C}(p)_{b a} \neq \emptyset$.

9. The hierarchical rule is monotone. Let profile pair $(p, q)$ forms an elementary change from $a b$ to $b a$. Let $R \in \varphi^{>}(p)_{b a}$. It is sufficient to prove that (i) $R \in \varphi^{>}(q)$ and (ii) $\varphi^{>}(q) \subseteq \varphi^{>}(p)$. As $(p, q)$ forms an elementary change we have the following three cases.

Case 1. $T_{p}=T_{q}$. Then $\widehat{T}_{p}=\widehat{T}_{q}$ and $\varphi^{>}(p)=\varphi^{>}(q)$. Therewith in this case (i) and (ii) follow.

Case 2. $T_{q}=T_{p} \cup\{b a\}$ and $a b, b a \notin T_{p}$. As $b a \in R$ and $\widehat{T}_{p} \subseteq R$, we have that $\widehat{T}_{p} \cup\{b a\}$ is acyclic. For a tournament $T$ let $\omega(T)=\sum_{x y \in T} \omega(x y)$. Then $\omega\left(\widehat{T}_{q}\right) \geq \omega\left(\widehat{T}_{p}\right)+\omega(b a)$. As $\widehat{T}_{q} \subseteq T_{q}$ it follows $\widehat{T}_{q} \backslash\{b a\} \subseteq T_{p}$. As $\widehat{T}_{q}$ is acyclic so is $\widehat{T}_{q} \backslash\{b a\}$. So, $\omega\left(\widehat{T}_{q} \backslash\{b a\}\right) \leq \omega\left(\widehat{T}_{p}\right)$. Hence, $\omega\left(\widehat{T}_{p}\right)+\omega(b a) \geq \omega\left(\widehat{T}_{q}\right)$. So, $\omega\left(\widehat{T}_{p}\right)+\omega(b a)=\omega\left(\widehat{T}_{q}\right)$. Because all weights are different powers of 2 this implies $\widehat{T}_{p} \cup\{b a\}=\widehat{T}_{q}$. But then $\widehat{T}_{q} \subseteq R$ which means that $R \in \varphi^{>}(q)$. Further, it follows from $\widehat{T}_{q}=\widehat{T}_{p} \cup\{b a\}$ that $\varphi^{>}(q)=\varphi^{>}(p)_{b a}$. Hence, $\varphi^{>}(q) \subset \varphi^{>}(p)$.

Case 3. $T_{p}=T_{q} \cup\{a b\}$ and $a b, b a \notin T_{q}$. Then $\widehat{T}_{q} \subseteq T_{q} \subseteq T_{p}$. As $\widehat{T}_{p} \subseteq R$ and $b a \in R$ it follows that $a b \notin \widehat{T}_{p}$. So, $\widehat{T}_{p} \subseteq T_{q}$. As $\widehat{T}_{p}$ is the best of $>$ among acyclic orders contained in $T_{p}$ it follows that $\omega\left(\widehat{T}_{p}\right) \geq \omega\left(\widehat{T}_{q}\right)$. As $\widehat{T}_{p} \subseteq T_{q}$ and $\widehat{T}_{q}$ is the best of $>$ among acyclic orders contained in $T_{q}$ it follows that $\omega\left(\widehat{T}_{p}\right) \leq \omega\left(\widehat{T}_{q}\right)$. The definition of $\omega$ implies $\widehat{T}_{p}=\widehat{T}_{q}$. But this means that $\varphi^{>}(p)=\varphi^{>}(q)$, which obviously implies (i) and (ii).

10. The hierarchical rule is tie-breaking. Let profile $(p, q)$ forms an elementary change from $a b$ to $b a$, with $\bar{p}_{a b}=\bar{p}_{b a}$. Let $\varphi^{>}(p)_{b a} \neq \emptyset$. It is sufficient to prove $\varphi^{>}(q)_{a b}=\emptyset$. Note that by the assumptions on $p$ and $q$ we have that $T_{q}=T_{p} \cup\{b a\}$ and $a b, b a \notin T_{p}$. As $\varphi^{>}(p)_{b a} \neq \emptyset$ Case 2 of the monotonicity proof of the hierarchical rule at point (9) above applies. There we prove that $\varphi^{>}(q)=\varphi^{>}(p)_{b a}$. As this means that $\varphi^{>}(q) \subseteq \mathbb{L}_{b a}$, it follows $\varphi^{>}(q)_{a b}=\emptyset$.

11. The hierarchical rule is not gradual. In Example 1, the profile pair $(p, q)$ forms an elementary change from $a b$ to $b a$. Further, $\bar{p}_{a b}=\bar{p}_{b a}=3, \varphi^{>}(p)=\{a c b\}$ and $\varphi^{>}(q)=\{c b a\}$. Hence, $\varphi^{>}(q)_{a b}=\emptyset$ and $\varphi^{>}(p)_{b a}=\emptyset$. This shows that $\varphi^{>}$is not gradual. 
Funding B. Can: This work is mostly financed by the Netherlands Organisation for Scientific Research (NWO) under the grant with Project No. 451-13-017 (VENI, 2014) and partially by Fonds National de la Recherche Luxembourg. The support of both institutes, therefore, is gratefully acknowledged. M. Pourpouneh: This work is supported by the Center for Blockchains and Electronic Markets (BCM) funded by the Carlsberg Foundation under Grant No. CF18-1112.

\section{Appendix}

\section{Monotonicity}

This appendix is on remarks related to update monotonicity.

A tournament choice correspondence, say $\Psi$, assigns to every tournament $T \in \mathbb{T}$ a subset $\Psi(T)$ of the set of alternatives $A$. For instance consider the Copeland tournament choice correspondence $\Psi^{C}$ defined for an arbitrary tournament $T$ as follows

$$
\Psi^{C}(T)=\{x \in A: \operatorname{Cscore}(x, T) \geq C \operatorname{Cscore}(y, T) \text { for all } y \in A\},
$$

where $\operatorname{Cscore}(x, T)=|\{z \in A: x z \in T\}|$.

In Brandt et al. (2016), the following monotonicity for such correspondences is introduced: a tournament choice correspondence is said to be monotone if for all $a \in A$ and all $T$ and $T^{\prime}$ in $\mathbb{T}$, with both (1) $T \cap(A \backslash\{a\})^{2}=T^{\prime} \cap(A \backslash\{a\})^{2}$ and (2) ay $\in T$ implies $a y \in T^{\prime}$ for all $y \in A \backslash\{a\}$

$$
a \in \Psi(T) \text { implies } a \in \Psi\left(T^{\prime}\right) .
$$

As the ranges of tournament preference correspondences differ from those of tournament choice correspondences, a direct comparison between update monotonicity and the monotonicity defined above is not possible. For a special subclass of tournament choice correspondences it is possible to translate the monotonicity condition to the setting of tournament preference correspondences. This translated condition appears to be weaker than update monotonicity. By that, for this subclass, the difference between monotonicity and update monotonicity can partially be revealed. Let $\Phi$ be a tournament preference correspondence. To $\Phi$ we can associate a tournament choice correspondence $\Psi^{\Phi}$ in the following way

$$
\Psi^{\Phi}(T)=\{x \in A: x \in \operatorname{best}(R) \text { for some } R \in \Phi(T)\} .
$$

Here $\operatorname{best}(R)=\{y \in A$ : for all $z \in A$ we have $y z \in R\}$. So, $\Psi^{\Phi}(T)$ chooses all best alternatives from the outcome of $\Phi$ at tournament $T$. It is obvious that $\Psi^{C}=\Psi^{\Phi^{C}}$.

Translating the monotonicity condition for tournament choice correspondences to tournament preference correspondences via such a best choice association yields the following. Let $\Phi$ be a tournament preference correspondence. We say that $\Phi$ is weakly monotone if for all tournament pairs $\left(T, T^{\prime}\right)$ forming an elementary change from $a b$ to $b a$, with $b \in \operatorname{best}(R)$ for some $R \in \Phi(T)$, there are $R^{\prime} \in \Phi\left(T^{\prime}\right)$ with $b \in \operatorname{best}\left(R^{\prime}\right)$. 
Proposition 1 Let $\Phi$ be a tournament preference correspondence and $\Psi^{\Phi}$ its associated tournament choice correspondence. Then

$\Phi$ is weakly monotone if and only if $\Psi^{\Phi}$ is monotone.

Proof (if part) Let $\Psi^{\Phi}$ be monotone. Let tournament pair $\left(T, T^{\prime}\right)$ forms an elementary change from $a b$ to $b a$, with $b \in \operatorname{best}(R)$ for some $R \in \Phi(T)$. It is sufficient to prove that $b \in \operatorname{best}\left(R^{\prime}\right)$ for some $R^{\prime} \in \Phi\left(T^{\prime}\right)$. As $b \in \operatorname{best}(R)$ for some $R \in \Phi(T)$, it follows that $b \in \Psi^{\Phi}(T)$. Monotonicity of $\Psi^{\Phi}$ implies that $b \in \Psi^{\Phi}\left(T^{\prime}\right)$. Meaning that for some $R^{\prime} \in \Phi\left(T^{\prime}\right)$ we have that $b \in \operatorname{best}\left(R^{\prime}\right)$.

(only if part) Let $\Phi$ be weakly monotone. Let tournament pair $\left(T, T^{\prime}\right)$ forms an elementary change from $a b$ to $b a$, with $b \in \Psi^{\Phi}(T)$. In order to prove that $\Psi^{\Phi}$ is monotone it is sufficient to show that $b \in \Psi^{\Phi}\left(T^{\prime}\right)$. As $b \in \Psi^{\Phi}(T)$, there are $R \in \Phi(T)$ with $b \in \operatorname{best}(R)$. Weak monotonicity of $\Phi$ guarantees the existence of $R^{\prime} \in \Phi\left(T^{\prime}\right)$ with $b \in \operatorname{best}\left(R^{\prime}\right)$. So, $b \in \Psi^{\Phi}\left(T^{\prime}\right)$.

To see that update monotonicity implies weak monotonicity take notations like in the definition of the latter notion. So, $R \in \Phi(T)_{b a}$. Update monotonicity implies that $\Phi(T)_{b a} \subseteq \Phi\left(T^{\prime}\right) \subseteq \Phi(T)$. In particular $R \in \Phi\left(T^{\prime}\right)$. So, we may take $R^{\prime}=R$ to conclude that $b \in \operatorname{best}\left(R^{\prime}\right)$. So, a consequence of Proposition 1 is that update monotonicity of $\Phi$ implies that $\Psi^{\Phi}$ is monotone. The Copeland preference correspondence $\varphi^{C}$ is not update monotone. Therefore, the Copeland tournament correspondence $\Phi^{C}$ is not T-monotone. The Copeland tournament correspondence $\Psi^{C}$ is monotone. Therefore, by Proposition $1, \Phi^{C}$ is weakly monotone, but not T-monotone.

\section{References}

Baigent N (1987) Preference proximity and anonymous social choice. Q J Econ 102(1):161-169

Baigent N, Klamler C (2004) Transitive closure, proximity and intransitivities. Econ Theory 23(1):175-181

Brandt F, Brill M, Harrenstein B (2016) Tournament solutions. In: Brandt F, Conitzer V, Endriss U, Lang J, Procaccia, AD (eds) Handbook of computational social choice (chap. 3)

Can B, Pourpouneh M, Storcken T (2018) Another re-characterization of Kemeny rule. Maastricht University Memo

Can B, Storcken T (2013) Update monotone preference rules. Math. Soc. Sci. 65(2):136-149

Can B, Storcken T (2018) A re-characterization of the Kemeny distance. J. Math. Econ. 79:112-116

Copeland AH (1951) A reasonable social welfare function. Technical report, mimeo, 1951. University of Michigan

Endriss U, de Haan R (2015) Complexity of the winner determination problem in judgment aggregation: Kemeny, Slater, Tideman, Young. In: Proceedings of the 2015 International Conference on Autonomous Agents and Multiagent Systems, pp 117-125

Fishburn PC (1977) Condorcet social choice functions. SIAM J Appl Math 33(3):469-489

Gibbard A (1973) Manipulation of voting schemes: a general result. Econometrica 41(4):587-601

Hudry O (2010) On the complexity of Slater's problems. Eur J Oper Res 203(1):216-221

Kemeny J (1959) Mathematics without numbers. Daedalus 88(4):577-591

Klamler C (2004) The Dodgson ranking and its relation to Kemeny's method and Slater's rule. Soc Choice Welf 23(1):91-102

Laffond G, Laslier J-F (1991) Slaters's winners of a tournament may not be in the Banks set. Soc Choice Welf 8(4):365-369 
McGarvey DC (1953) A theorem on the construction of voting paradoxes. Econom J Econom Soc 608-610

Meskanen T, Nurmi H (2006) Distance from consensus: A theme and variations. In: Mathematics and democracy, Springer, Berlin, pp 117-132

Miller NR (1980) A new solution set for tournaments and majority voting: further graph-theoretical approaches to the theory of voting. Am J Political Sci 24(1):68-96

Monsuur H, Storcken T (1997) Measuring intransitivity. Math Soc Sci 34(2):125-152

Moulin H (1986) Choosing from a tournament. Soc Choice Welf 3(4):271-291

Muller E, Satterthwaite MA (1977) The equivalence of strong positive association and strategy-proofness. J Econ Theory 14(2):412-418

Nurmi H (2002) Measuring disagreement in group choice settings. In: Holler MJ, Kliemt H, Schmidtchen, Streit ME (eds) Power and Fairness. Jahrbuch für Neue Politische Ökonomie, pp 20

Satterthwaite MA (1975) Strategy-proofness and arrow's conditions: Existence and correspondence theorems for voting procedures and social welfare functions. J Econ Theory 10(2):187-217

Slater P (1961) Inconsistencies in a schedule of paired comparisons. Biometrika 48(3/4):303-312

Young HP (1975) Social choice scoring functions. SIAM J Appl Math 28(4):824-838

Young HP, Levenglick A (1978) A consistent extension of Condorcet's election principle. SIAM J Appl Math 35(2):285-300

Publisher's Note Springer Nature remains neutral with regard to jurisdictional claims in published maps and institutional affiliations. 